\title{
Embedded nanomicro syringe on chip for molecular therapy
}

This article was published in the following Dove Press journal:

International Journal of Nanomedicine

17 November 201।

Number of times this article has been viewed

\section{Muhammad Arif Jalil' \\ Nathaporn Suwanpayak ${ }^{2,3}$ \\ Kathawut Kulsirirat ${ }^{3}$ \\ Saisudawan Suttirak ${ }^{3}$ \\ Jalil $\mathrm{Ali}^{4}$ \\ Preecha PYupapin ${ }^{3}$}

'Ibnu Sina Institute of Fundamental Science Studies, Nanotechnology

Research Alliance, Universiti

Teknologi Malaysia, Johor Bahru,

Malaysia; ${ }^{2}$ King Mongkut's Institute

of Technology Ladkrabang,

Chumphon Campus, Chumphon,

Thailand; ${ }^{3}$ Nanoscale Science

and Engineering Research Alliance,

Advanced Research Center

for Photonics, Faculty of Science,

King Mongkut's Institute

of Technology Ladkrabang,

Bangkok, Thailand; ${ }^{4}$ Institute

of Advanced Photonics Science,

Nanotechnology Research Alliance,

Universiti Teknologi Malaysia,

Johor Bahru, Malaysia
Correspondence: Preecha P Yupapin

Nanoscale Science and Engineering Research Alliance, Advanced Research Center for Photonics, Faculty of Science, King Mongkut's Institute of Technology Ladkrabang, Bangkok 10520, Thailand

Tel +66 23298414

Fax +66 23298410

Email kypreech@kmitl.ac.th
Background: A novel nanomicro syringe system was proposed for drug storage and delivery using a PANDA ring resonator and atomic buffer. A PANDA ring is a modified optical add/drop filter, named after the well known Chinese bear. In principle, the molecule/drug is trapped by the force generated by different combinations of gradient fields and scattering photons within the PANDA ring. A nanomicro needle system can be formed by optical vortices in the liquid core waveguide which can be embedded on a chip, and can be used for long-term treatment. By using intense optical vortices, the required genes/molecules can be trapped and transported dynamically to the intended destinations via the nanomicro syringe, which is available for drug delivery to target tissues, in particular tumors. The advantage of the proposed system is that by confining the treatment area, the effect can be decreased. The use of different optical vortices for therapeutic efficiency is also discussed.

Keywords: nanomicro syringe, nanomicro needle, molecular therapy, therapeutic efficiency, cancer

\section{Introduction}

Optical trapping is one of the techniques that can be used to manipulate living cells, ${ }^{1}$ and is the most powerful single-molecule technique for cellular application in vivo. ${ }^{2}$ Nowadays, optical tweezers are used in many fields of physical, chemical, and biological science. Optical tweezers have provided a deeper insight into the dynamic process responsible for cell motion in biology. ${ }^{3}$ Hosokawa et $\mathrm{al}^{4}$ have demonstrated optical trapping of synaptic vesicles in a hippocampal neuron and found that intracellular synaptic vesicles can be trapped at the focal spot during laser irradiation. A molecular network using the blood circulation for a drug delivery system was designed by Mitatha et al. ${ }^{5}$ A combination of optical trapping forces and router network systems has been used to control the blood circulation in capillary networks and blood quality in human organs. Optical forces can be used to manipulate biological and mesoscopic particles in a noncontact manner. This is the foundation of the trapped particle as a revolutionary tool for single-molecule biophysics. ${ }^{6}$

A single red blood cell deformability test has been performed by optical trapping of plastic in a microfluidics chip ${ }^{7,8}$ and a lab-on-a-chip for red blood cell transportation in the capillary network to circulate oxygen and carbon dioxide throughout the human body. ${ }^{9}$ Optical trapping for manipulation of molecules in liquid core capillaries and its application to drug delivery have been reported by Suwanpayak et al. ${ }^{11}$ A PANDA ring resonator was used to form, transmit, and receive a microscopic volume of drug by controlling the ring parameters. A microscopic volume can be trapped and 
moved dynamically within the wavelength router or network. Promising techniques to trap and transport a microscopic volume within the add/drop multiplexer have been reported theoretically ${ }^{6}$ and experimentally ${ }^{11}$ using optical tweezers. Static tweezers are now well recognized and widely used. The optical tweezer generation technique has become a powerful tool for manipulation of micrometer-sized particles. Dynamic tweezers are now also used in practical work ${ }^{12-14}$ involving viruses, living cells, and subcellular organelles, as well as translocation of DNA and DNA-protein complexes. Another area of interest is the measurement of elastic properties of cell membranes, long strands of single DNA molecules, and filamentous proteins. ${ }^{15-18}$

Schulzyz et al have shown that transfer of trapped atoms between two optical potentials can be performed. ${ }^{19}$ Optical tweezers use the forces exerted by intensity gradients in strongly focused beams of light to trap and move microscopic volumes of matter. Other combinations of forces are induced by the interaction between photons, caused by photon scattering effects. In practice, the field intensity can be adjusted and tuned to produce the desired gradient field and a scattering force to produce a suitable trapping force. Hence, the appropriate force can be configured for the transmitter/ receiver parts, which can perform long-distance microscopic transportation.

Using the proposed system, gene therapy is done by copying of a gene into the specific target for treatment of a disease.${ }^{17}$ By using a PANDA ring resonator, the generated optical potential wells can be used to transport and move nonpathogenic gene/molecular matter. ${ }^{18}$ The drug molecules can be trapped and transported within the blood capillary network, in which the atoms/molecules of drugs can be stored and transported in a hollow liquid core waveguide by using add/drop optical vortices as in liquid core microneedles (nanomicro syringe), where the required drug types can be controlled/released into targets by a single ring resonator (syringe). During operation, the dynamic optical tweezers/ vortices are generated using dark soliton, bright soliton, and a Gaussian pulse propagating within an add/drop optical multiplexer incorporating nanoring resonators (PANDA ring resonator), in which the trapped molecules can be moved and transported into specific target diseases, such as tumors, cancers, and abnormal cells. The dynamic behavior of soliton and the Gaussian pulse has been well described by Kachynski et al. ${ }^{20}$ The design of the original PANDA ring structure and its application in drug delivery was reported by Oguchi and Terada. ${ }^{21}$ The parameters of the fabricated device are as close as possible to those of the original design. The present study investigated the use of two different wavelength tweezers, molecular buffers, and bus networks to transport volumes of drug, especially larger ones.

In principle, trapping forces are exerted by the intensity gradients of highly focused light beams which trap and transport microscopic volumes of matter. The optical forces are customarily defined by the relationship between optical scattering force and gradient force $\left(\mathrm{F}_{\text {grad }}\right){ }^{22}$ In the Rayleigh regime, since the electromagnetic field is uniform across the dielectric, the trapping forces decompose naturally into two components. Thus, the particles can be treated as induced point dipoles. An increase in the numerical aperture increases the gradient strength due to a decrease in focal spot size ${ }^{23}$ which can be formed within the tiny system, for instance, a nanoscale device (nanoring resonator). The different optical trapping force structures are compared in terms of static and dynamic tweezers, as shown in Figure 1. In this case, the dynamic tweezers (gradient fields) can be in the forms of bright solitons, Gaussian pulses, or dark solitons, which can be used to trap the required microscopic volume, where there are four different center wavelengths of tweezers generated. ${ }^{24,25}$

The power output $\left(P_{t 1}\right)$ at the through port is written as

$$
P_{t 1}=\left|E_{t 1}\right|^{2} \text {. }
$$

The power output $\left(P_{t 2}\right)$ at the drop port is

$$
P_{t 2}=\left|E_{t 2}\right|^{2}
$$

In the system proposed here, the trapping force is produced using a Gaussian pulse and bright soliton, where the valley of the dark soliton is generated and controlled within the PANDA ring resonator by the control port signals. Figure 2 shows the output field $\left(E_{t 1}\right)$ at the through port. In the add/drop device, the nonlinear refractive index is ignored because it does not affect the system. The add/drop device is silicon-based and linear, where the nonlinear effect occurs due to the PANDA ring resonator, in which the slight change of phase delay has no effect and can be ignored. The electric fields, $E_{0}$ and $E_{0 \mathrm{~L}}$, are the circulating fields within the nanoring at the right and left side of the add/drop optical filter. To form the broad spectrum output, two nonlinear ring resonators are introduced to form a proposed PANDA ring structure, named after the well known Chinese bear, and a term used to describe the core fiber structure which maintains polarization. In this work, the proposed PANDA ring resonator is a modified add/drop filter. By using a broad-spectrum output, multitweezers can be generated and used to trap and transport molecules via the proposed system, in which the nanomicro needle-shaped 


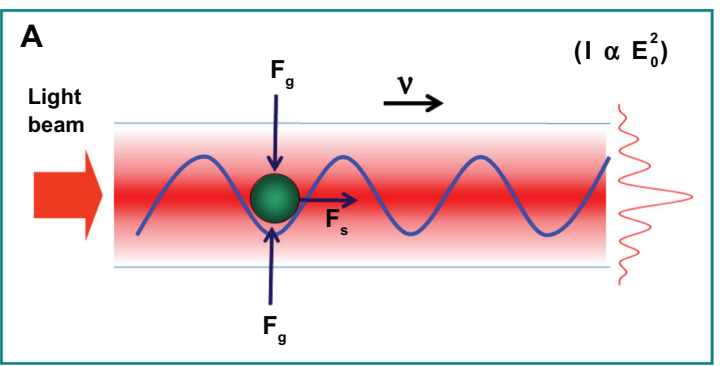

Static tweezer

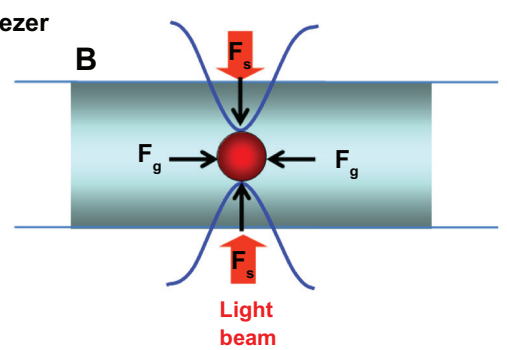

Dynamics tweezer
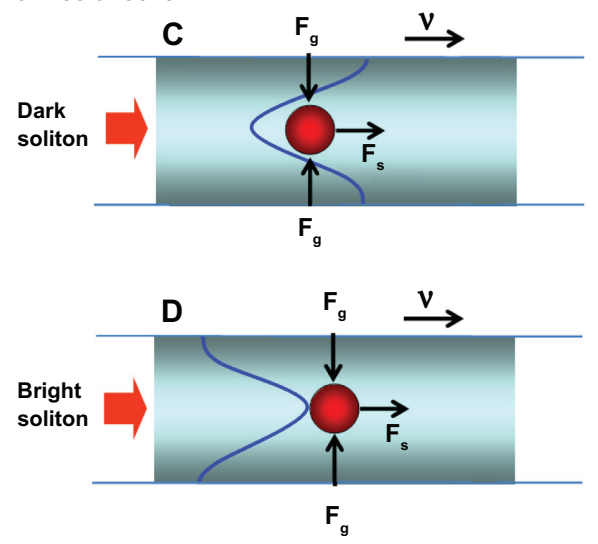

Figure I Different forms of dynamic and static optical tweezers.

injection chip operates in the same way as a syringe, and can be used for tumor-specific therapy.

\section{Molecular therapy using an embedded nanomicro syringe}

Figure 3 shows a schematic diagram of an embedded microchip device as well as the relevant molecular therapeutic details.
Optical trapping force can be used to trap atoms, molecules, and living cells and is measured on a pico $\left(10^{-2}\right)$ Newton scale. Input data are gathered for the entrapped drug, which can be delivered using the nanosyringe and reach the desired target specifically. The proposed molecular buffer of living cells can be transported through microchannels via a throughput port $\left(\mathrm{E}_{t 1}\right)$ on the PANDA ring resonator. Molecules moving in a nanocapillary can be blocked by an interaction between the capillary and molecules. In this proposed system, the optical trappings are induced by an optical gradient, and scattering forces become potential optical wells which transport atoms and molecules in a liquid channel. A molecular buffer is required to be included in the system, which plays an important role in storing or delaying atoms/molecules over a period of time, which gives enough time for operation. ${ }^{26,27}$ A molecular buffer is a new device, which is operated in the same way as a gas buffer. ${ }^{28}$ In this case, it is assumed that the spherical particle is polystyrene $(\mathrm{n}=1.5894)$ and the liquid medium is water $(n=1.33)$. The required optical power that can be used to trap particles of a certain size/polarizability is $9.1 \mathrm{~W}$, more details on which can be found elsewhere. ${ }^{29}$ In simulation, a dark/bright soliton, a Gaussian pulse in the 1.3-1.7 $\mu \mathrm{m}$ wavelength, peak power $1 \mathrm{~W}$, and a pulse width of $35 \mathrm{fs}$ is fed into the system via the input port, where the coupling coefficients are $\kappa_{0}=0.5, \kappa_{1}=0.35, \kappa_{2}=0.1$, and $\kappa_{3}=0.35$. The ring radii are $\mathrm{R}_{\text {add }}=20 \mu \mathrm{m}, \mathrm{R}_{\mathrm{R}}=\mathrm{R}_{\mathrm{L}}=5 \mu \mathrm{m}$ Evidence for a practical device with a radius of $2-3 \mu \mathrm{m}$ has been reported by Zhu et al. ${ }^{25}$ In this case, the dynamic tweezers (gradient fields) are in the form of bright solitons, Gaussian pulses, and dark solitons, which are used to trap the required microscopic volume. In this investigation, four tweezers with different wavelength centers are generated, the dynamic movements of which are shown in Figure 4, where Figure 4A represents tweezers with different sizes and wavelengths and Figure 4B represents tunable tweezers coupling constant variations. The required drug volumes are obtained at the drop port outputs.
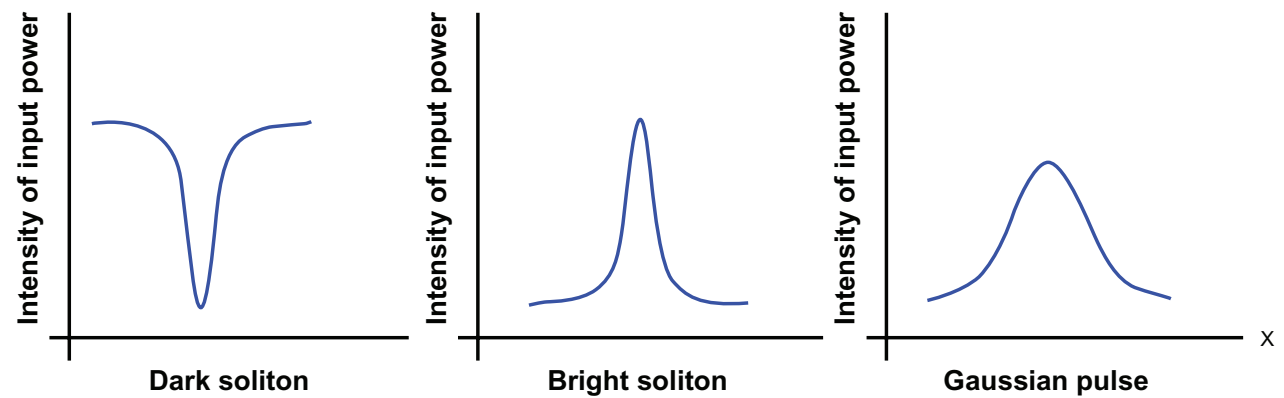

Figure 2 Different input light signals. 

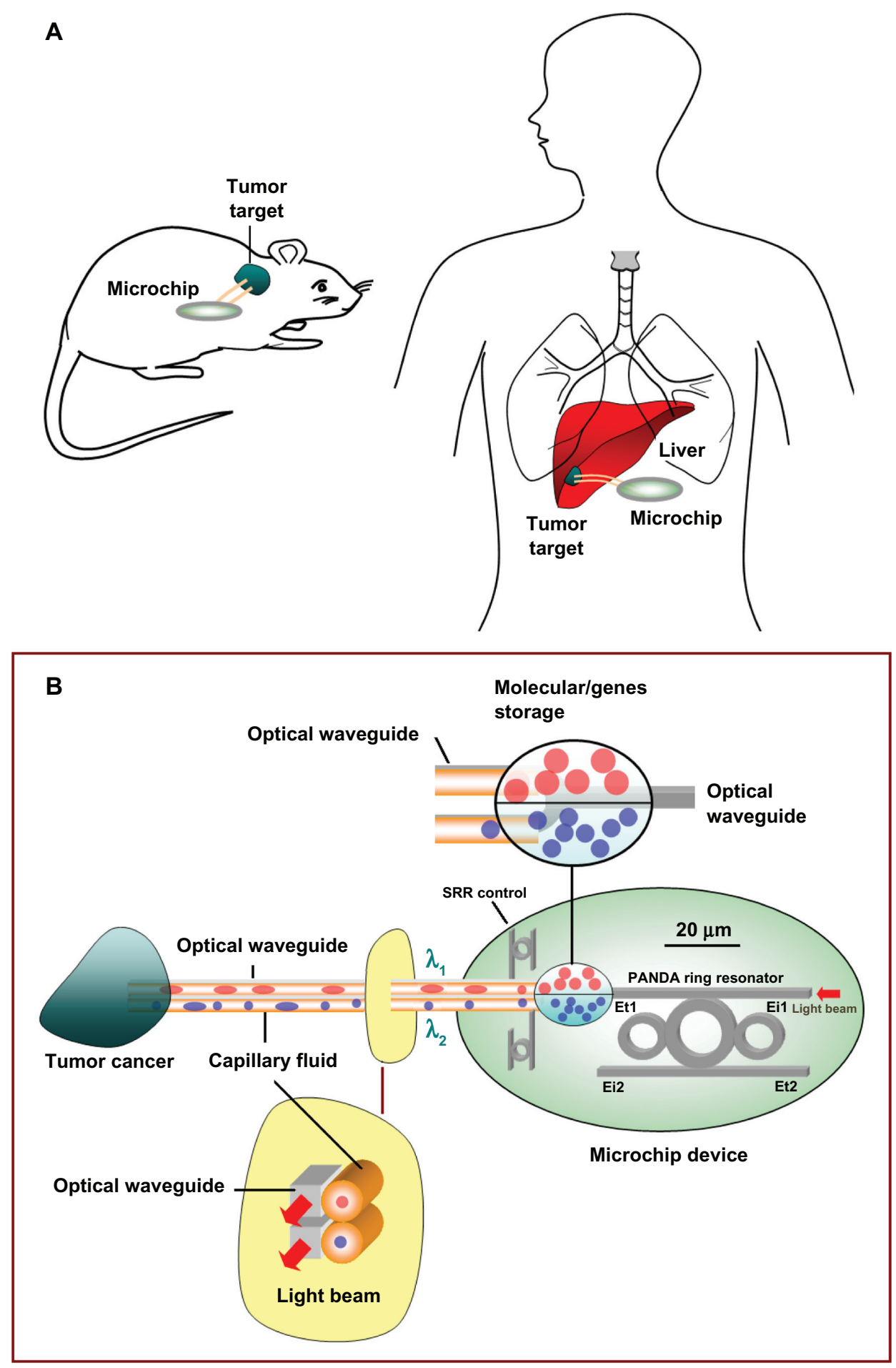

Figure 3 Schematic diagram of embedded microchip device and molecular therapeutic details.

In practice, the fabrication parameters can be easily controlled by the ring resonator radii instead of the coupling constants. The optical coupling coefficients represent the fraction of light transmission through the coupler, while the loss coefficients represent the fraction of light loss into the ring. ${ }^{30}$ An important feature of this system is that the tunable tweezers can be obtained by tuning (controlling) the add (control) port input signal, in which the required number of microscopic volumes (atoms, photons, molecules) can be obtained at the drop/through ports, otherwise they propagate within the PANDA ring without collapsing or decaying into the waveguide. One of the advantages of the 

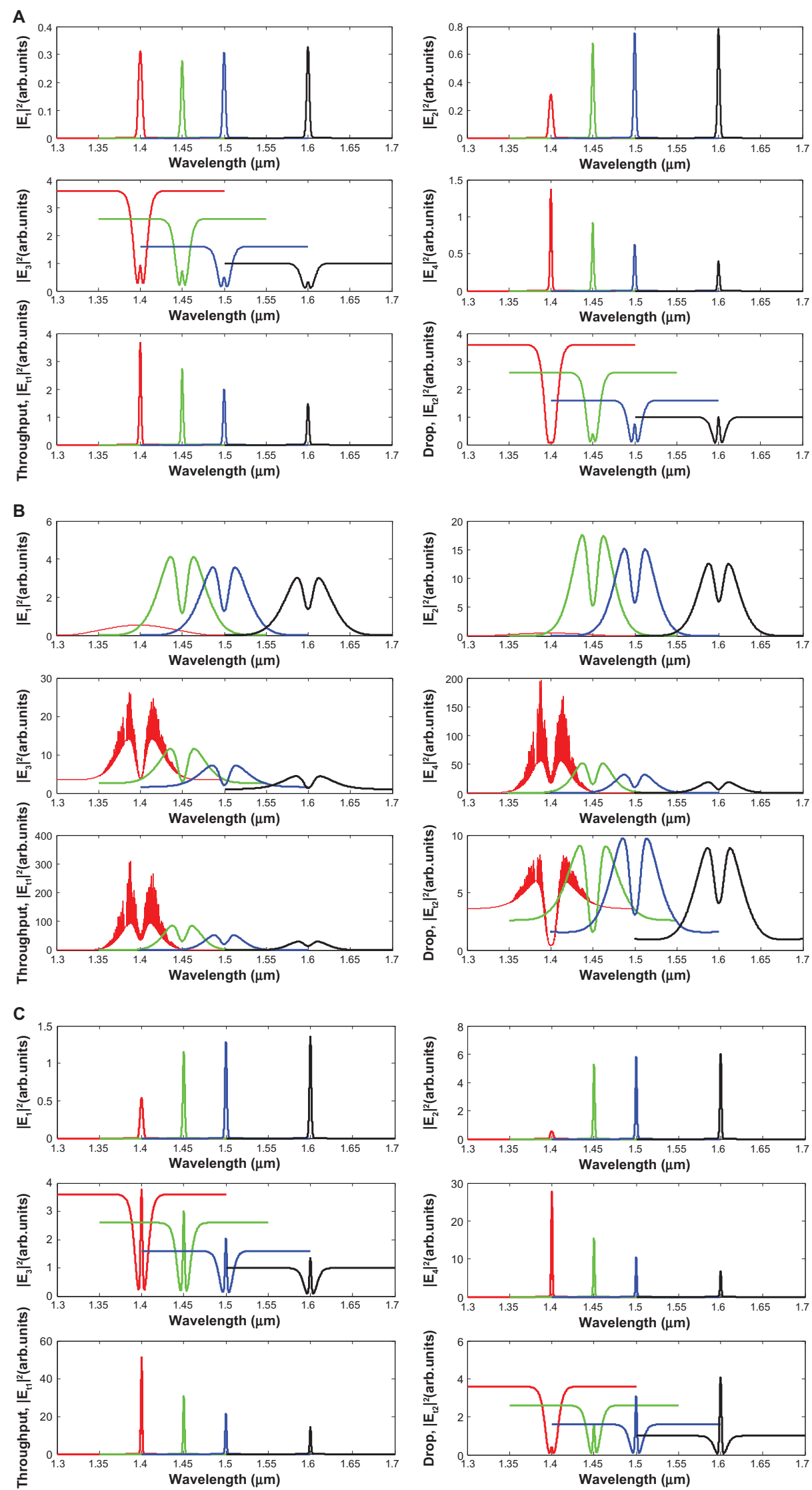

Figure 4 Generated trapping tools with different input powers, where (A) Gaussian pulse and dark soliton, (B) Gaussian pulse, and bright soliton and (C) dark soliton and bright soliton. $R_{a d}=20 \mu \mathrm{m}, R_{R}=R_{L}=5 \mu \mathrm{m}$. The coupling coefficients are $\kappa_{0}=0.5, \kappa_{1}=0.35, \kappa_{2}=0.1$ and $\kappa_{3}=0.35$. 

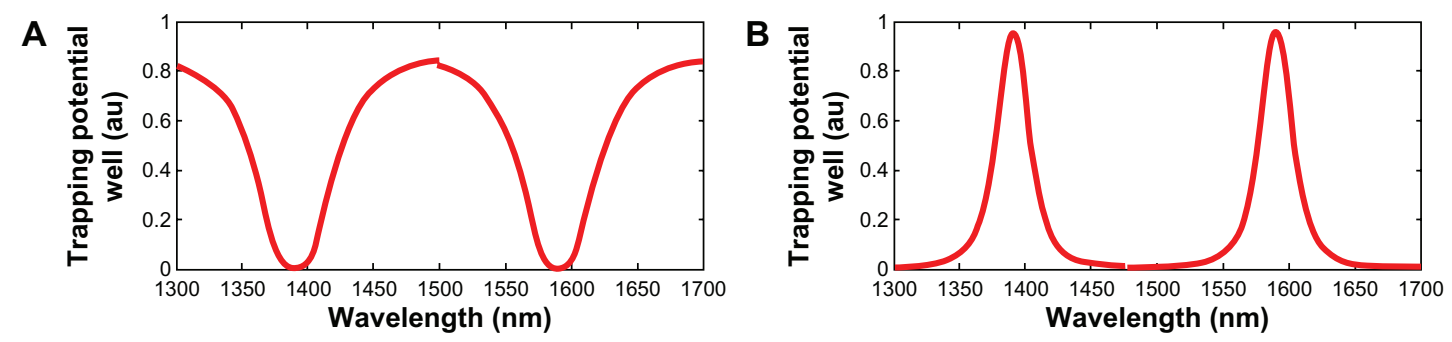

Figure 5 Results of the normalized potential wells with the wavelength centers are $1400 \mathrm{~nm}$ and $1600 \mathrm{~nm}$, where $R_{a d}=20 \mu \mathrm{m}, R_{R}=R_{L}=5 \mu m$. The coupling coefficients are $\kappa_{0}=0.5, \kappa_{1}=0.35, \kappa_{2}=0.1$, and $\kappa_{3}=0.35$.

proposed system is that the transmitter and receiver can be fabricated on-chip and alternatively can be operated by a single device. The magnitude of optical trapping force is in the pico Newton range, depending on the relative refractive index of the particle. ${ }^{31}$ The particle radius located in the cavity decreases with reduction in the refractive indices compared with the host medium. ${ }^{32}$ The molecular trapping probe can be adjusted to select a drug molecule size of $80 \mathrm{~nm}$ or $0.2681 \times 10^{-3} \mu \mathrm{m}^{3}$ per potential well (Figure 5A and B), but the fluidic microscopic volume can be transported faster, depending on the viscosity of both media and particle..$^{33}$ The molecular trapping probe can be used for drug molecule transport at the through port and networks, the parameters for which are given in Figure 5. The proposed system provides multiple accesses to the drug volumes and targets.

Generally speaking, tumors are similar to organs, with unique physiology giving rise to an unusual set of drug delivery barriers. Cancer and tumor therapies are limited by nonuniform drug delivery via blood vessels, where the stored drug is transported by blood into regions of the tumor and distributed to region by vascular network. ${ }^{34}$ Recently, a therapeutic problem in tumors has been resolved, in which the convective drug transport from blood vessels into and throughout most of the tumor tissue is realized. The modular design of the carrier facilitates the exchange of different targeting moieties and small interfering RNAs to permit its use in a variety of tumor types. An ideal carrier protects small interfering RNA from exogenous nucleases, prolongs the systemic half-life, and promotes preferential uptake into diseased tissues. Tumor cells often overexpress transferrin receptors on their surfaces, and this protein has also been studied as a ligand for tumor-targeted delivery. ${ }^{34,35}$

Molecular or gene therapy is a great advance in our understanding of how to manipulate genes and has set the stage for scientists to alter a patient's genetic material to fight or prevent disease ${ }^{36}$ However, gene therapy may have both positive $\mathrm{e}^{36-38}$ and negative ${ }^{38}$ effects when used as a medical tool. In this experiment, we designed a useful tool for medical therapy and health care, which includes genetic manipulation of DNA or RNA in a patient's cells to fight disease. In practice, the optimization of molecular therapy to treat disorders requires the development of a system specific for the targeted disease process. ${ }^{37}$ Further discussion of molecular therapy can be found elsewhere. ${ }^{39,40}$

\section{Conclusion}

We have proposed an interesting system that can be used for molecular diagnosis and therapy, ${ }^{41}$ in which the treatment area, especially tumor tissue, can be confined and optimized. The trapped living cells/drug can be transported into the liquid core channels/capillaries via specifically designed optical tweezers and waveguides, in which drug molecules/ cells can be trapped, stored, and delivered to specific targets. Such a system can be fabricated on a chip and used as a drug injection tool (syringe) for targeted treatment to cancerous tissue. However, the size of the drug molecule is shown to be varied by the character of the interacting light used, ie, a standing wave versus a propagating wave. ${ }^{18}$ The design of these molecular diagnostic and drug delivery agents for biomedical application is summarized, where specific conditions for a wide range of targets are introduced and compared with antibodies, ${ }^{35}$ in which cells/drug particles could be manipulated for drug delivery to tumor cells/target via optical vortices. The advantage of the proposed system is that the confined treatment area can give less effect during the treatment, which will be the subject of our continuing research and investigation.

\section{Acknowledgments}

We would like to thank the Institute of Advanced Photonics Science, Nanotechnology Research Alliance, Universiti Teknologi Malaysia, and King Mongkut's Institute of 
Technology Thailand for providing their research facilities. This research work has been supported by a Tier 1/Flagship research grant from the Universiti Teknologi Malaysia, MyBrain 15 Fellowship/Ministry of Higher Education SLAB Fellowship, and a Ministry of Higher Education research grant. NS would like to acknowledge the King Mongkut's Institute of Technology for partial support in higher education.

\section{Disclosure}

The authors report no conflicts of interest in this work.

\section{References}

1. Ashkin A. The study of cells by optical trapping and manipulation of living cells using infrared laser beams. ASGSB Bull. 1991;4: 133-146.

2. Bao G, Suresh S. Cell and molecular mechanics of biological materials. Nat Mater. 2003;2:715-725.

3. Ashkin A. Optical trapping and manipulation of neutral particles using lasers. Proc Natl Acad Sci U S A. 1997;94:4853-4858.

4. Hosokawa C, Kudoh SN, Kiyohara A, Taguchi T. Optical trapping of synaptic vesicles in neurons. Appl Phys Lett. 2011;98:163705-1-3.

5. Mitatha S, Moongfangklang N, Jalil MA, et al. Multi-access drug delivery network and stability. Int J Nanomed. 2011;6:1757-1764.

6. Dholakia K, Cizmar T. Shaping the future of manipulation. Nat Photonics. 2011;5:335-341.

7. Kohles SS, Liang Y, Saha AK. Volumetric stress-strain analysis of optohydrodynamically suspended biological cells. J Biomech Eng. 2011;133:011004-011006.

8. Obrist D, Weber B, Buck A, Jenny P. Red blood cell distribution in simplified capillary networks. Philos Transact A Math Phys Eng Sci. 2010;368:2897-2918.

9. Lee WG, Park K, Bang H, et al. Single red blood cell deformability test using optical trapping in plastic microfluid chip. Proceedings of the 31 annual international IEEE EMBS Special Topic on Conference Microtechnologies in Medicine and Biology, Kahuku, Oahu, Hawaii, May $12-15,2005$.

10. Chen YC, Chen GY, Lin YC, Wang GJ. A lab-on-a-chip capillary network for red blood cell hydrodynamics. Microfluid Nanofluidics. 2010;9:585-591.

11. Suwanpayak N, Jalil MA, Teeka C, Ali J, Yupapin PP. Optical vortices generated by a PANDA ring resonator for drug trapping and delivery applications. Biomed Opt Express. 2011;2:159-168.

12. Oguchi K, Terada S. Optical network system, optical router, fault recovery method of optical network, and program. Patent JP 2010063009 2010.

13. Piyatamrong B, Kulsirirat K, Mitatha S, Yupapin PP. Dynamic potential well generation and control using double resonators incorporating in an add/drop filter. Mod Phys Lett B. 2010;24:3071-3082.

14. Cai H, Poon A. Optical manipulation and transport of microparticle on silicon nitride microring resonator-based add-drop devices. Opt Lett. 2010;35:2855-2857.

15. Otto O, Steinbock LJ, Wong DW, Gornall JL, Keyser UF. Note: Direct force and ionic-current measurements on DNA in a nanocapillary. Rev Sci Instrum. 2011;82:086102.

16. Coirault C, Pourny JC, Lambert F, Lecarpentier Y. Optical tweezers in biology and medicine. Med Sci (Paris). 2003;19:364-367. French.

17. Rice J, Ottensmeier CH, Stevenson FK. DNA vaccines: precision tools for activating effective immunity against cancer. Nat Rev Cancer. $2008 ; 8: 108-120$
18. Egashira K, Terasaki A, Kondow T. Photon-trap spectroscopy applied to molecules adsorbed on a solid surface: Probing with a standing wave versus a propagating wave. Appl Opt. 1998;80:5113-5115.

19. Schulzyz M, Crepaz H, Schmidt-Kaler F, Eschner J, Blatt R. Transfer of trapped atoms between two optical tweezer potentials. J Mod Opt. 2007;54:1619-1626.

20. Kachynski AV, Kuzmin AN, Pudavar HE, Kaputa DS, Cartwright AN, Prasad PN. Measurement of optical trapping forces by use of the two-photon-excited fluorescence of microspheres. Opt Lett. 2003;28:2288-2290.

21. Oguchi K, Terada S. Optical network system, optical router, fault recovery method of optical network, and program. Patent JP 2010063009. 2010.

22. Svoboda K, Block SM. Biological applications of optical forces. Ann Rev Biophy Biomol Struct. 1994;23:247-282.

23. Teeka C, Jalil MA, Yupapin PP, Ali J. Novel tunable dynamic tweezers using dark-bright soliton collision control in an optical add/drop filter. IEEE Trans Nanobiosci. 2010;9:258-262.

24. Juan ML, Righini M, Quidant R. Plasmon nano-optical tweezers. Nat Photonics. 2011;5:349-356.

25. Zhu J, Ozdemir SK, Xiao YF, Li L, He L, Chen DR. On-chip single nanoparticle detection and sizing by mode splitting in an ultrahigh- $Q$ microresonator. Nat Photonics. 2010;4:46-49.

26. Bauer WR, Nadler W. Molecular transport through channels and pores: Effects of in-channel interactions and blocking. Proc Natl Acad Sci US A. 2006;103:11446-11451.

27. Rosenberry MA, Reyes JP, Tupa D, Gay TJ. Radiation trapping in rubidium optical pumping at low buffer-gas pressures. Phys Rev A 2007;75:023401-1- 6 .

28. Lignie MC, Woerdman JP. Light-induced drift of Na in molecular buffer gases. J Phys B: At Mol Opt Phys. 1990;23:417-426.

29. Suwanpayak N, Jalil MA, Aziz MS, Ali J, Yupapin PP. Molecular buffer using a PANDA ring resonator for drug delivery use. Int $J$ Nanomed. 2011;6:575-580

30. McKinnon WR, Xu DX, Storey C, et al. Extracting coupling and loss coefficients from a ring resonator. Opt Express. 2009;17:18971-18982.

31. Waggoner PS, Palmer JS, Antonov VN, Weaver JH. Metal nanostructure growth on molecular buffer layers of $\mathrm{CO}_{2}$. Surf Sci. 2005;596:12-20.

32. Fischer M, Sørensen KB. Calibration of trapping force and response function of optical tweezers in viscoelastic. J Opt A: Pure Appl Opt. 2007;79:239-250

33. Chauhan VP, Stylianopoulos T, BoucherY, Jain RK. Delivery of molecular and nanoscale medicine to tumors: Transport barriers and strategies. Annu Rev Chem Biomol Eng. 2011;2:281-298.

34. Palanca-Wessels MC, Convertine AJ, Cutler-Strom R, et al. Anti-CD22 antibody targeting of $\mathrm{pH}$-responsive micelles enhances small interfering RNA Delivery and gene silencing in lymphoma cells. Mol Ther. 2011;19:1529-1537.

35. Ikeda Y, Taira K. Ligand-targeted delivery of therapeutic siRNA. Pharm Res. 2006;23:1631-1640.

36. West J, Rodman DM. Gene therapy for pulmonary diseases. Chest. 2001;119:613-617.

37. Barlow-Stewart K, Burnett L. Ethical considerations in the use of DNA for the diagnosis of diseases. Clin Biochem Rev. 2006;27:53-61.

38. Kawabata K, Tashiro K, Sakurai F, et al. Positive and negative regulation of adenovirus infection by CAR-like soluble protein, CLSP. Gene Ther. 2007; 14:1199-1207.

39. National Cancer Institute. Gene therapy for cancer. Available from: http://www.cancer.gov/cancertopics/factsheet/Therapy/gene. Accessed September 25, 2011.

40. Leiden JM. Human gene therapy: The good, the bad, and the ugly. Circ Res. 2000;86:923-925.

41. Lee JH, Yigit MV, Mazumdar D, Lua Y. Molecular diagnostic and drug delivery agents based on aptamer-nanomaterial conjugates. $A d v$ Drug Deliv Rev. 2010;62:592-605. 


\section{Publish your work in this journal}

The International Journal of Nanomedicine is an international, peerreviewed journal focusing on the application of nanotechnology in diagnostics, therapeutics, and drug delivery systems throughout the biomedical field. This journal is indexed on PubMed Central, MedLine, CAS, SciSearch $\AA$, Current Contents ${ }^{\circledR} /$ Clinical Medicine,
Journal Citation Reports/Science Edition, EMBase, Scopus and the Elsevier Bibliographic databases. The manuscript management system is completely online and includes a very quick and fair peer-review system, which is all easy to use. Visit http://www.dovepress.com/ testimonials.php to read real quotes from published authors.

Submit your manuscript here: http://www.dovepress.com/international-journal-of-nanomedicine-journal 